\title{
Severely underestimated rate of wind speed decline jeopardizes China's carbon peak emission target
}

\section{Shuanglei Feng ( $\square$ fengsl@epri.sgcc.com.cn )}

Renewable Energy Center, China Electric Power Research Institute https://orcid.org/0000-0003-45846467

\section{Weisheng Wang}

Renewable Energy Center, China Electric Power Research Institute https://orcid.org/0000-0002-42894079

\section{Zongpeng Song}

Renewable Energy Center, China Electric Power Research Institute https://orcid.org/0000-0002-1331$002 X$

\section{Qing Yang}

Huazhong University of Science and Technology https://orcid.org/0000-0003-4251-554X

\section{Feng Liu}

Department of Electrical Engineering, Tsinghua University

\section{Yunhe Hou}

Shenzhen Institute of Research and Innovation, The University of Hong Kong

\section{Zheng Wang}

Renewable Energy Center, China Electric Power Research Institute

\section{Bo Wang}

Renewable Energy Center, China Electric Power Research Institute

\section{Shengwei Mei}

Department of Electrical Engineering, Tsinghua University

\section{Article}

Keywords:

Posted Date: January 20th, 2022

DOI: https://doi.org/10.21203/rs.3.rs-1135115/v1

License: (c) (i) This work is licensed under a Creative Commons Attribution 4.0 International License. Read Full License 


\title{
Severely underestimated rate of wind speed decline jeopardizes China's carbon peak emission target
}

Shuanglei Feng ${ }^{1,2,8^{*}}$, Weisheng Wang ${ }^{1,2,8^{*}}$, Zongpeng Song ${ }^{1,2^{*}}$, Qing Yang ${ }^{3}$, Feng Liu ${ }^{4}$, Yunhe $\mathrm{Hou}^{5,6}$, Zheng Wang ${ }^{1,2}$, Bo Wang ${ }^{1,2}$, Shengwei Mei ${ }^{4,7}$

${ }^{1}$ Renewable Energy Center, China Electric Power Research Institute, Peking, China. ${ }^{2}$ State Key Laboratory of Operation and Control of Renewable Energy \& Storage Systems, Peking, China. ${ }^{3}$ School of Energy and Power Engineering, Huazhong University of Science and Technology, Wuhan, Hubei, China. ${ }^{4}$ Department of Electrical Engineering, Tsinghua University, Peking, China. ${ }^{5}$ Department of Electrical and Electronic Engineering, The University of Hong Kong, Hong Kong, China. ${ }^{6}$ Shenzhen Institute of Research and Innovation, The University of Hong Kong, Shenzhen, China. ${ }^{7}$ Qinghai Key Lab of Efficient Utilization of Clean Energy (TusInstitute for Renewable Energy, Qinghai University), Xining, Qinghai, China. ${ }^{8}$ These authors contributed equally: Shuanglei Feng, Weisheng Wang. *e-mail: fengsl@epri.sgcc.com.cn; wangws@epri.sgcc.com.cn; songzongpeng@epri.sgcc.com.cn.

\begin{abstract}
:
The development of wind energy is indispensable in the pursuit of global carbon neutrality. Following decades of climate change, China's annual average wind speed has shown a clear decline, but the rate of this decline and its potential impacts on the need for wind power development in China have not been quantified. Here, we reveal
\end{abstract}


that China's observed wind speed has declined significantly at $-0.169 \mathrm{~m} / \mathrm{s} / 10 \mathrm{yr}, 33.33$ times the rate predicted by the Coupled Model Intercomparison Project (CMIP) of World Climate Research Programme, indicating a severe underestimation by those models. We attribute this underestimation to CMIP neglecting the atmospheric boundary layer height and greatly underestimating the Arctic amplification effect on wind speed. Scenario analyses demonstrate that China's future wind power installed capacity, investment costs, and land area occupied based on the observed trend scenario will increase by approximately 53\% compared to any trend of three (high, medium, and low) CMIP emission scenarios in order to meet China's wind-generated electricity target and carbon peak emission goal in 2030. Hence, formulating its wind energy development plans based on these CMIP scenarios' trends will prevent China from meeting its low-carbon electricity generation of carbon peak emission target by 2030 and delaying the 2060 goal of carbon neutrality, emphasizing that the CMIP models urgently need to be improved. These findings should serve as a warning to countries throughout the Northern Hemisphere to formulate wind power development plans in consideration of the climate change impacts in the pursuit of global carbon neutrality.

\section{Introduction}

The International Renewable Energy Agency (IRENA) predicted that ${ }^{1}$ in order to achieve the $1.5^{\circ} \mathrm{C}$ temperature rise goal of the Paris Agreement, more than one-third of global electricity generation in 2050 will need to derive from wind; as a result, wind energy will become the world's largest source of electricity power. At present, China 
already has the largest installed capacity and the fastest growth rate of wind power worldwide; at the end of 2020, China's installed wind power capacity reached $37.8 \%$ of the global total ${ }^{2}$, and its capacity is set to grow further. According to the plan for achieving China's carbon peak emission target by $2030^{3}, 25.9 \%$ of all electricity consumed in 2030 will be provided by non-hydro renewable energy ${ }^{4}$. Assuming that half of its electricity will be provided by wind power, China's wind power generation potential in 2030 will be more than twice that in $2020^{5}$. The development of wind power in China will be decisive for realizing carbon neutrality globally.

Nevertheless, the scale of wind power development that would be required to satisfy China's national strategy depends heavily on future wind speed levels. Yet, a decline of wind speed ${ }^{6}$ due to global climate change in the north hemisphere might have a profound impact on wind power development and even global carbon neutrality. At present, Coupled Model Intercomparison Project $(\mathrm{CMIP})^{7}$ climate data are widely used to predict future climate trends ${ }^{8-10}$, including those of wind speed ${ }^{6,11-13}$. However, past research on the uncertainty and accuracy of CMIP models has typically focused on temperature and precipitation, etc. ${ }^{14-16}$, rather than on wind speed. Hence, the reliability of CMIP wind speed predictions and the consequences of errors in those predictions for future wind power development remain unclear.

In this paper, we aim to quantify the rates of wind decline in different speed ranges by observed data in China, and then to identify if CMIP models could predict wind speed correctly in the past, finally to evaluate its potential impacts on the future need for wind power development in China. To this end, by collecting observed wind speed 
data from 771 ground stations throughout China during 1981-2014, we reveal a longterm decline in the average wind speed that CMIP remarkably underestimates. Our theoretical analysis indicates that this underestimation may be due to CMIP neglecting the atmospheric boundary layer height and greatly underestimating the Arctic amplification effect on wind speed. Finally, comparing our observed wind speed trend and the three CMIP scenarios of high, medium, and low emissions, we estimate the new installed capacity, new investment costs, and new land area occupation required to fulfil China's 2030 wind energy generation goal according to the national carbon emission reduction strategy. The scenario differences demonstrate the potential impacts of underestimating the wind speed rate of decline to China's wind power development goal and how this threat jeopardizes the progress made toward the national carbon peak emission goal. This research is of great significance for facilitating China's carbon emission reduction and carbon neutrality strategies and further provides a scientific basis for formulating wind power development plans under the meteorological influences of global climate change. Our findings should also help other countries at similar latitudes in the Northern Hemisphere formulate wind power development plans more reasonably.

\section{The long-term trend of observed wind speeds in China}

For this study, we collected long-term (1981-2014) observation data from surface meteorological stations throughout China. A total of 771 stations were checked to guarantee that they had never been moved and that the data were continuous. Considering that the height of a typical modern-day wind turbine is $80 \mathrm{~m}$, the original 
wind speeds at a height of $10 \mathrm{~m}$ (10 m wind speeds) were extrapolated to a height of 80 $\mathrm{m}$ (80 $\mathrm{m}$ wind speeds) using a power law relation. The annual average wind speed among all 771 stations is plotted in Figure 1. The stations are divided into three wind speed intervals $(0-3,3-6$, and $6-9 \mathrm{~m} / \mathrm{s})$ according to the annual averages over the $34-$ yr study period. All wind speed averages, and more than $75 \%$ of all stations show a declining trend (i.e., have a negative linear fitting coefficient; Table 1). In addition, the rates of decline appear to increase, and are observed at more stations, with increasing wind speed interval.
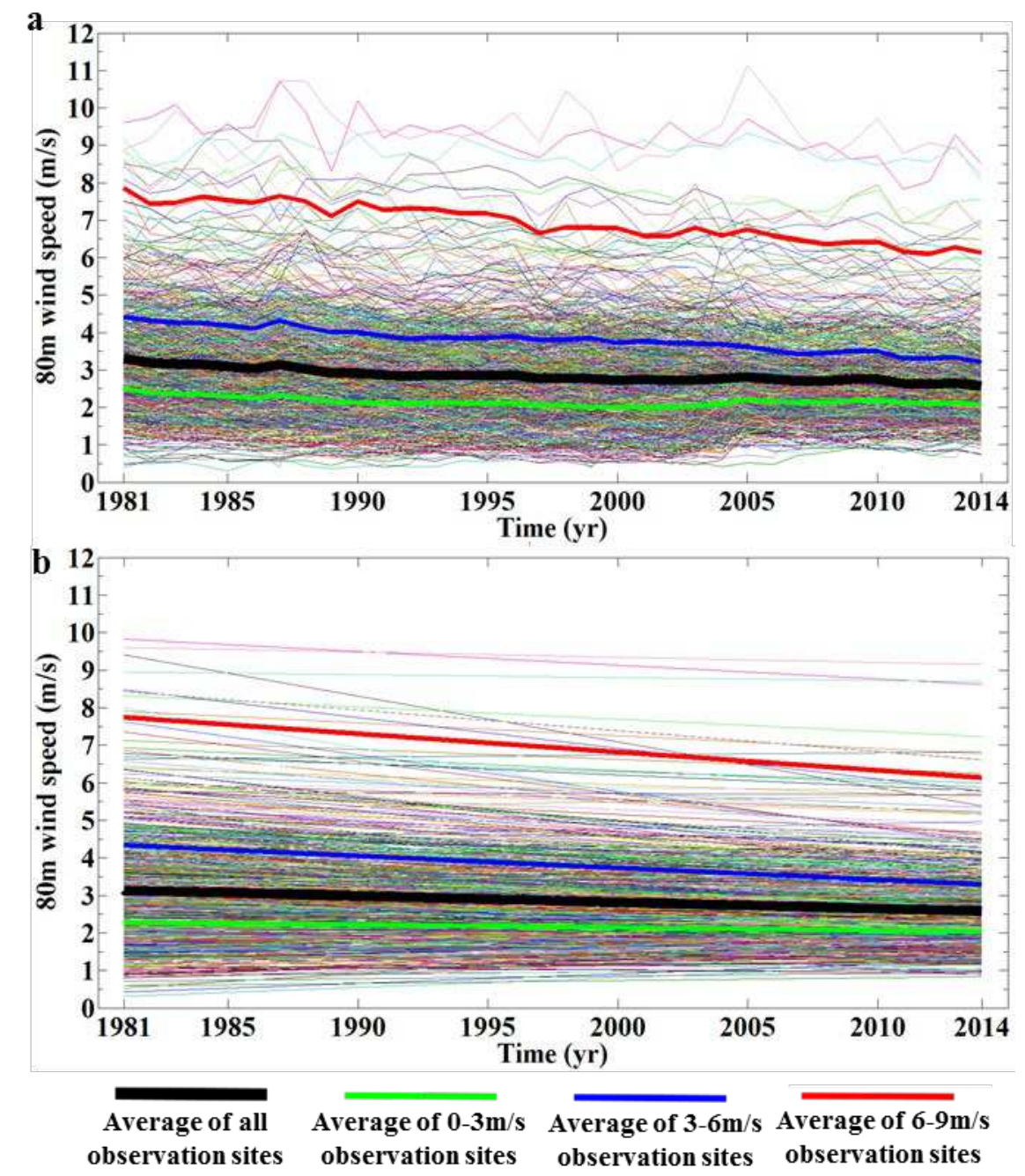

Figure 1 | Observed $80 \mathrm{~m}$ wind speeds averaged over 1981-2014. a, Time series of the annual average wind speeds. Each thin line represents the annual average wind speed at an 
individual observation station, and the thick lines represent the averages for all stations (black) and for the stations in the different wind speed intervals (colours). The 4 windspeed averages all show declining trends, with higher wind speeds exhibiting more significant declining trends. $b$, Linear fitting results of the annual average wind speeds.

As shown in Table 1, the wind speed rate of decline among all stations during 1981-2014 was $-0.169 \mathrm{~m} / \mathrm{s} / 10 \mathrm{yr}$. At wind speeds above $3 \mathrm{~m} / \mathrm{s}$ and up to $6 \mathrm{~m} / \mathrm{s}$, the decline rate was $-0.316 \mathrm{~m} / \mathrm{s} / 10 \mathrm{yr}$, and more than $93 \%$ of the stations yielded a rate of decline. Furthermore, at wind speeds between 6 and $9 \mathrm{~m} / \mathrm{s}$, the decline rate reached $0.488 \mathrm{~m} / \mathrm{s} / 10 \mathrm{yr}$.

Table 1 | The average rate of decline among all 771 observation stations and the proportions of stations with rates of decline in different wind speed intervals. A station experiencing a decline is defined as having a linear fitting coefficient of less than 0 over the 34 -yr period.

\begin{tabular}{|c|c|c|c|}
\hline Station group & $\begin{array}{c}\text { Total } \\
\text { number } \\
\text { of stations }\end{array}$ & $\begin{array}{l}\text { Number (proportion) of } \\
\text { stations with a rate of } \\
\text { decline }\end{array}$ & $\begin{array}{l}\text { Decline rate } \\
\text { (m/s/10 yr) }\end{array}$ \\
\hline All stations & 771 & $585(75.9 \%)$ & -0.169 \\
\hline $\begin{array}{l}\text { Stations with } 34-\mathrm{yr} \text { average wind } \\
\text { speed }>0 \text { and }<3 \mathrm{~m} / \mathrm{s}\end{array}$ & 480 & $314(65.4 \%)$ & -0.074 \\
\hline $\begin{array}{l}\text { Stations with } 34-\mathrm{yr} \text { average wind } \\
\text { speed }>3 \text { and } \leq 6 \mathrm{~m} / \mathrm{s}\end{array}$ & 274 & $255(93.1 \%)$ & -0.316 \\
\hline $\begin{array}{l}\text { Stations with } 34-y r \text { average wind } \\
\text { speed }>6 \text { and } \leq 9 \mathrm{~m} / \mathrm{s}\end{array}$ & 15 & $14(93.3 \%)$ & -0.488 \\
\hline
\end{tabular}

\section{Analysing the mechanism responsible for the declining wind speeds}

Next, we theoretically analysed the mechanism responsible for the declining wind speeds in China by using the classic geostrophic wind relationship in the free 
atmosphere, the Ekman spiral relationship of the atmospheric boundary layer, and the ideal gas state equation, etc. We derived the following long-term theoretical relationship of the $80 \mathrm{~m}$ wind speed $V_{80 m}$ :

$$
V_{80 m}=-\frac{R}{f} \frac{\partial T_{2 m}}{\partial y}\left[1+e^{-\frac{160 \pi}{H}}-2 e^{-\frac{80 \pi}{H}} \cos \left(\frac{80 \pi}{H}\right)\right]^{\frac{1}{2}}
$$

where $-\frac{\partial T_{2 m}}{\partial y}$ is the $2 \mathrm{~m}$ height latitudinal temperature $\left(T_{2 m}\right)$ gradient of latitudes $(y)$, reflecting the Arctic amplification (the Arctic warming pace has been approximately double that of global average in recent decades) effect ${ }^{17-19}, H$ is the height of the local atmospheric boundary layer, $R$ is the ideal gas constant, and $f$ is the geostrophic parameter. Equation 1 illustrates that the latitudinal temperature gradient $-\frac{\partial T_{2 m}}{\partial y}$ (the horizontal influencing factor) and the atmospheric boundary layer height $H$ (the vertical influencing factor) are the two key parameters affecting the wind speed trend. However, this long-term declining wind speed trend is not restricted to China but is applicable to all other countries at similar latitudes in the Northern Hemisphere.

To verify the above theoretical equation and quantitatively explore the influences of $-\frac{\partial T_{2 m}}{\partial y}$ and $H$, we used the European Centre for Medium-Range Weather Forecasts (ECMWF) Reanalysis v5 (ERA5) dataset ${ }^{20}$ to compute the theoretical wind speed. ERA5, which contains spatiotemporal grid data that can replace splattering observations, offers the most refined reanalysis data currently available, with a horizontal resolution of $0.25^{\circ} \times 0.25^{\circ}$. We extracted $-\frac{\partial T_{2 m}}{\partial y}$ and $H$ from the ERA5 grid data corresponding to the 771 observation stations and then calculated the theoretical $V_{80 m}$ and its rate of decline at each site. The theoretical declining trend reaches -0.124 $\mathrm{m} / \mathrm{s} / 10 \mathrm{yr}$, which is very close to the observed rate of $-0.169 \mathrm{~m} / \mathrm{s} / 10 \mathrm{yr}$, as shown in 
Figure 2.

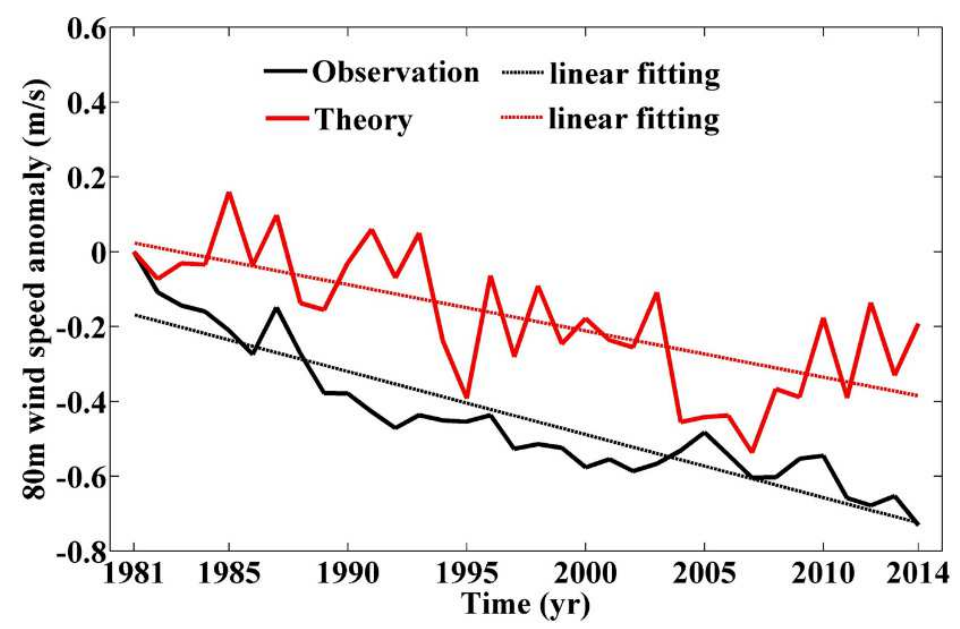

Figure 2 | Comparison between the theoretical and observed long-term wind speed trends of all 771 observation stations' average . To compare the trends, the figure reflects only the wind speed anomaly, that is, with the wind speed in 1981 subtracted. The theoretically derived declining trend is very close to that of the observation, which verifies the correctness of the theoretical wind speed equation.

To better understand the magnitudes of $-\frac{\partial T_{2 m}}{\partial y}$ and $H$, their long-term trends are plotted in Figure 3. It can be seen that $-\frac{\partial T_{2 m}}{\partial y}$ presents a long-term declining trend caused by the Arctic amplification effect, which leads to a decrease in wind speed according to Equation 1. This north-south temperature difference is the driving force of atmospheric circulation, and thus, a decrease in circulation corresponds to a decline in wind speed.

In contrast, $H$ presents a long-term increasing trend, which also leads to a decrease in wind speed. The long-term change in $H$ is affected mainly by the local surface temperature, surface roughness, etc. In particular, surface warming raises $H$. As $H$ increases, the upper free atmosphere (associated with high wind speeds with strong momentum) above the atmospheric boundary layer moves away from the surface, 
increasing the distance over which momentum is transmitted (between high altitudes and the surface) and thus decreasing the surface wind speed.

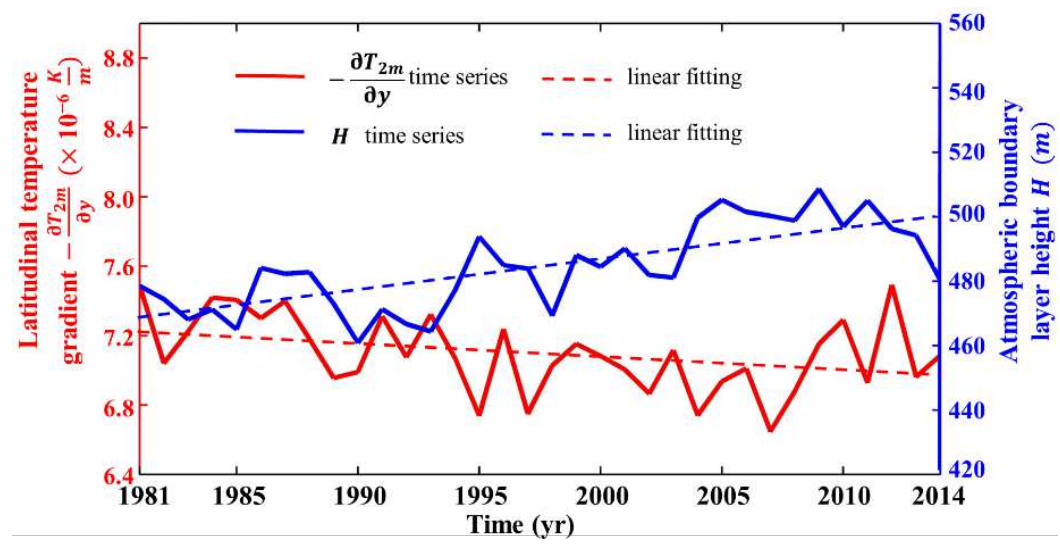

Figure 3 | Long-term average trends of $-\frac{\partial T_{2 m}}{\partial y}$ and $H$ at 771 observation stations in China from the ERA5 data. - $\frac{\partial T_{2 m}}{\partial y}$ shows a decreasing trend, while $H$ shows an increasing trend. According to our theoretical analysis of the long-term wind speed trend (Equation 1), a decrease in $-\frac{\partial T_{2 m}}{\partial y}$ and an increase in $H$ would both cause the wind speed to decrease.

Next, we calculated the individual contributions of $-\frac{\partial T_{2 m}}{\partial y}$ and $H$ to the rate of wind speed decline . First, we considered only a change in $-\frac{\partial T_{2 m}}{\partial y}$, keeping $H$ constant at the value obtained for 1981 , and we obtained a $-0.057 \mathrm{~m} / \mathrm{s} / 10 \mathrm{yr}$ rate of decline. Then, we considered only a change in $H$, this time keeping $-\frac{\partial T_{2 m}}{\partial y}$ constant at the value obtained for 1981 , and we obtained a $-0.074 \mathrm{~m} / \mathrm{s} / 10 \mathrm{yr}$ rate of decline. These calculation results indicate that the influences of $-\frac{\partial T_{2 m}}{\partial y}$ and $H$ are approximately similar, although the influence of $H$ is slightly greater.

\section{Evaluating the CMIP-predicted wind speeds in China}

Currently, wind speed trends are predicted mainly based on CMIP climate models. For example, by using CMIP data, researchers have evaluated the future trends of wind speed and wind energy for the Northern Hemisphere ${ }^{6}$, including Europe ${ }^{11}$, China ${ }^{12-13}$ 
and India ${ }^{13}$.

To evaluate the CMIP wind speed predictions, we selected the latest release, CMIP Phase 6 (CMIP6) ${ }^{21}$ for an analysis of three future scenarios from among the CMIP shared socioeconomic pathways (SSPs) ${ }^{22}$ : the low-emission SSP126 scenario, the medium-emission SSP245 scenario, and the high-emission SSP585 scenario.

Figure 4 plots both the predicted wind speed anomalies obtained by various CMIP6 models (using the annual averaged time series beginning in 1981 from the 771 ground observation stations in each CMIP6 model and extending to 2060) and the observed wind speed anomaly (1981-2014). To better compare the wind speed trends among the different models, only the wind speed anomalies are plotted. For the CMIP6 models with historical simulation data, the anomaly was calculated relative to that recorded in 1981; for the CMIP6 models without historical simulation data, the anomaly was calculated relative to the value in 2015 . 

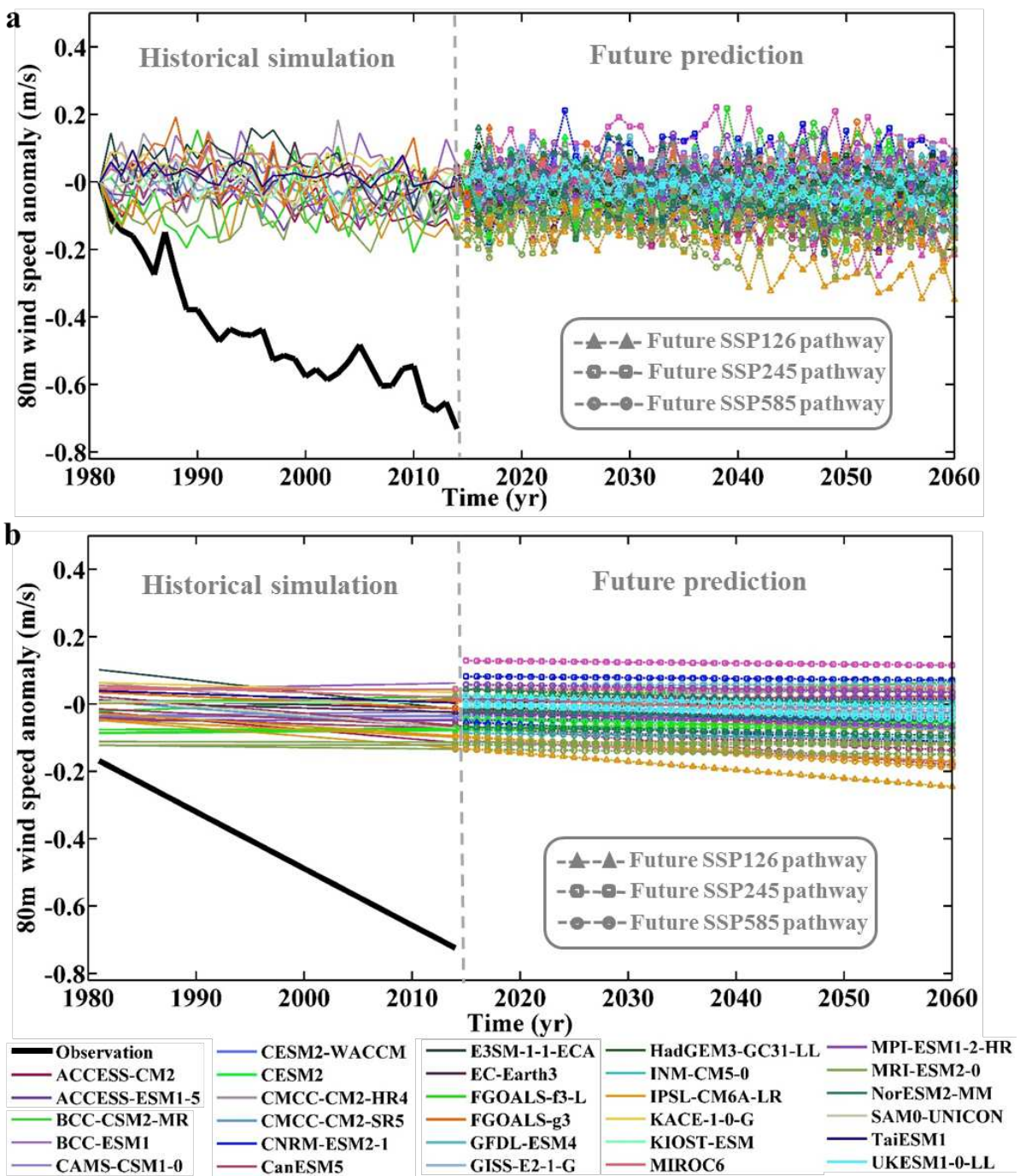

Figure 4 | Observed and CMIP-predicted wind speed anomalies. a, Time series of

the annual average wind speed. Each thin line represents the anual average for a CMIP6 model (each incorporating the data from 771 observation stations). In this figure, the CMIP6 models severely underestimate the wind speed rate of decline. b, Linear fitting result of the annual average wind speed.

We found that all CMIP6 models severely underestimate the rate of decline of the wind speed anomaly over the observation period. The average rate of decline in SSP126, $\mathrm{SSP} 245$, and SSP585 is $-0.0015 \mathrm{~m} / \mathrm{s} / 10 \mathrm{yr},-0.0037 \mathrm{~m} / \mathrm{s} / 10 \mathrm{yr}$, and $-0.0059 \mathrm{~m} / \mathrm{s} / 10 \mathrm{yr}$, respectively (for the rate of decline of each model, see Supplementary Tables 2-4). As such, even the highest-emission scenario (SSP585) has a rate of decline of only 
approximately $3 \%$ of the rate that was obtained from the observations $(-0.169 \mathrm{~m} / \mathrm{s} / 10$ yr).

We attribute the underestimation associated with the CMIP models to two limitations of those models. First, on the basis of our theoretically derived equation to calculate the $80 \mathrm{~m}$ wind speed (Equation 1), we hypothesize that a major potential source of this underestimation is their neglect of the atmospheric boundary layer height $H$; therefore, the effect of long-term changes in $H$ on the wind speed anomaly cannot be modelled. Second, the modelled rates of decline are much smaller than even the theoretical rate obtained by considering only the contribution of $-\frac{\partial T_{2 m}}{\partial y}$; that is, the CMIP models greatly underestimate the Arctic amplification effect on wind speed.

The external boundary and forcing conditions constitute the main difference between the CMIP predictions and historical models. As shown above, the CMIP models ignore the atmospheric boundary layer height and underestimate the Arctic amplification effect on historical rate of decline. Therefore, the CMIP models are highly likely to underestimate China's future rate of decline. Moreover, the equation for calculating the long-term $80 \mathrm{~m}$ wind speed trend is applicable to other mid-latitude countries in the Northern Hemisphere. Consequently, CMIP will suffer from the same severe underestimation problem when making predictions for those nations.

Calculating the wind power development scale corresponding to China's 2030 carbon peak emission target under different scenarios

Then, we conducted a comparative analysis of the wind power installed capacity, investment costs, and area of new land occupation among the future scenarios to assess 
the effects of the underestimation in the decline in wind speed that we found to arise from the use of the CMIP models on China's plans for achieving its 2030 carbon peak emission target. First, we assessed the 2030 wind speed under the four scenarios (observed, and the three CMIP model scenarios) and we used this to estimate the wind power installed capacity in 2030 . Next, we compared this installed capacity prediction for 2030 with the installed capacity in 2020 , and finally, we converted this predicted new installed capacity expansion to new investment costs and new land occupation.

Scenario 1 follows the linear rate of decline of observed wind speeds in China (hereinafter referred to as the observation trend scenario). We applied the same rate of decline of the wind speed from 2015 to 2030 as for 1981 to 2014. Although the observation trend scenario may be biased, it is based on the statistically significant linear fit of the long-term observation data and is thus an extension of the actual longterm trend. Scenario 1 therefore represents the future trend as a serious possibility. Meanwhile, Scenarios 2, 3, and 4 follow the linear rates of decline of the CMIP low-, medium- and high-emission scenarios, respectively, where the rate of decline in each scenario is the average of all CMIP models under the corresponding emission scenario.

This analysis focuses on the wind speed employed to generate wind power. Hence, we first obtained the annual average wind speed $v_{\text {ave,2014 }}$ employed to generate wind power in the reference year (2014) based on China's wind-generated electricity in 2014, China's installed capacity in 2014, the typical statistical Rayleigh distribution of wind speed (see Figure 6a in Methods ), and the typical power curve of a $2 \mathrm{MW}$ wind turbine (see Figure $6 \mathrm{~b}$ in Methods). The calculated wind speeds under the 4 scenarios from 
2014 to 2030 are plotted in Figure 5, and the proposed method to calculate the $80 \mathrm{~m}$ wind speed is detailed in the Methods.

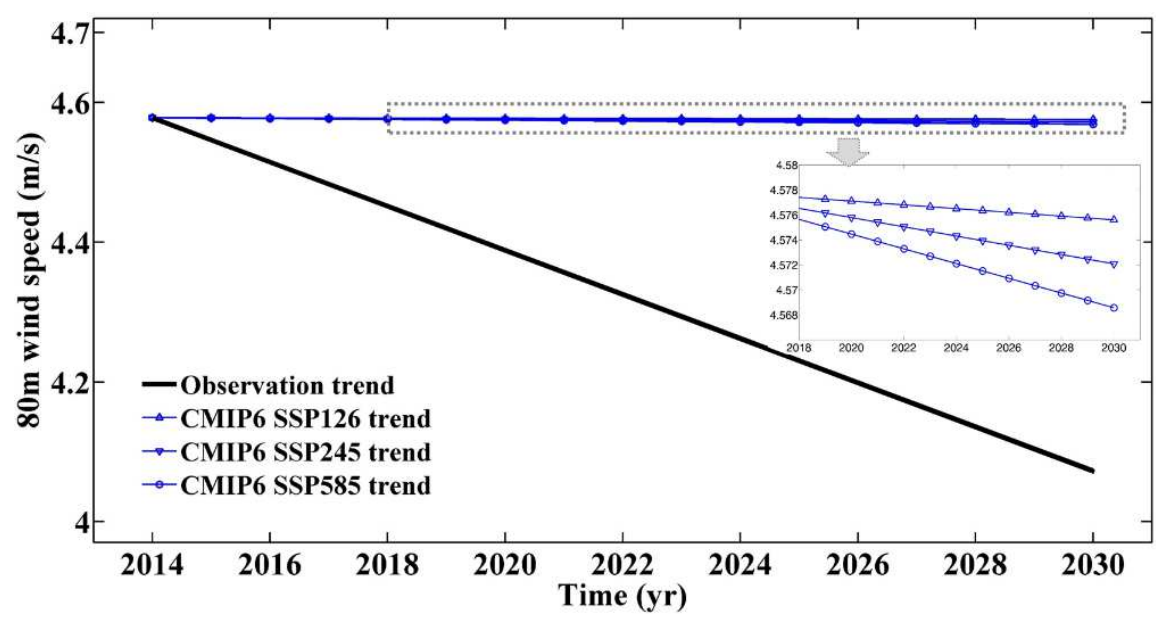

Figure 5 | Annual average wind speeds from 2014 to 2030 under the extrapolated observation trend scenario and the CMIP high-, mediumand low-emission scenarios.

Next, we calculated the wind power installed capacity in 2030 based on the annual average wind speed $v_{\text {ave,2030, }}$, the planned amount of wind-generated electricity by China in $2030\left(1.425 \times 10^{12} \mathrm{~kW}\right.$-h according to China's 2030 plan $^{4}$ to generate $50 \%$ of all power by non-hydro renewable energy), the typical statistical Rayleigh wind speed distribution, and the typical power curve of a $2 \mathrm{MW}$ wind turbine. Then, China's installed capacity in 2030 was compared to that in $2020^{5}$. Using this comparison together with the investment costs and land occupation area of a typical $2 \mathrm{MW}$ wind turbine, we obtained the total new investment costs and new area of land occupation in 2030 relative to the values in 2020, as shown in Table 2.

Table 2 | Calculated installed capacity, investment costs, and area of land occupation in 2030 under the CMIP high-, medium- and low-emission scenarios and the observation trend scenario. The values in the observation trend scenario each represent an increase of at least $52 \%$ compared to those in the three CMIP scenarios. 


\begin{tabular}{|c|c|c|c|c|}
\hline & Scenario & 2030 & $\begin{array}{l}\text { Increment } \\
\text { relative to } \\
\qquad 2020\end{array}$ & $\begin{array}{l}\text { Proportion of } \\
\text { increment relative to } \\
\text { the CMIP6 SSP126 } \\
\text { scenario }\end{array}$ \\
\hline \multirow{4}{*}{$\begin{array}{l}\text { Installed } \\
\text { capacity } \\
\left(10^{4} \mathrm{~kW}\right)\end{array}$} & CMIP6 SSP126 trend & 82500 & 54347 & - \\
\hline & CMIP6 SSP245 trend & 82700 & 54547 & $0.37 \%$ \\
\hline & CMIP6 SSP585 trend & 82850 & 54697 & $0.64 \%$ \\
\hline & Observation trend & 111300 & 83147 & $52.99 \%$ \\
\hline \multirow{4}{*}{$\begin{array}{l}\text { Investment } \\
\text { costs }\left(10^{8}\right. \\
\text { USD) }\end{array}$} & CMIP6 SSP126 trend & - & 5798 & - \\
\hline & CMIP6 SSP245 trend & - & 5819 & $0.37 \%$ \\
\hline & CMIP6 SSP585 trend & - & 5835 & $0.64 \%$ \\
\hline & Observation trend & - & 8871 & $52.99 \%$ \\
\hline \multirow{4}{*}{$\begin{array}{l}\text { Land area } \\
\text { occupied } \\
\left(\mathrm{km}^{2}\right)\end{array}$} & CMIP6 SSP126 trend & - & 53912 & - \\
\hline & CMIP6 SSP245 trend & - & 54111 & $0.37 \%$ \\
\hline & CMIP6 SSP585 trend & - & 54259 & $0.64 \%$ \\
\hline & Observation trend & - & 82482 & $52.99 \%$ \\
\hline
\end{tabular}

We found that the new installed capacity, new investment costs, and new land occupation in 2030 vs. 2020 of CMIP's three scenarios were essentially the same as each other (to within 0.01 percentage points; Table 2). However, the observation trend scenario will increase by approximately $53 \%$ compared to the three CMIP scenarios. Specifically, under the observation trend scenario, the new installed capacity is predicted to exceed $2.8 \times 10^{8} \mathrm{~kW}$, the new investment costs are expected to pass $3.0 \times 10^{11}$ USD, and the new area of land occupation is anticipated to span more than $28,000 \mathrm{~km}^{2}$. The above results show that the CMIP models' underestimation of the wind speed rate of decline will jeopardize China's wind power development strategy that is being 
implemented to achieve the carbon peak emission target by 2030; accordingly, this discrepancy will certainly delay the goal of reaching national carbon neutrality by 2060 .

\section{Conclusion}

In this study, we analysed wind speed observation data during 1981-2014 from 771 meteorological stations and simulation data from numerous CMIP models, and revealed that these models severely underestimate the wind speed rate of decline in China. The theoretical derivation of an equation for computing the $80 \mathrm{~m}$ wind speed suggests two main reasons for the declining wind speed trend, that is, a decreasing latitudinal temperature gradient and an increasing atmospheric boundary layer height. Specifically, the CMIP models neglect to account for the atmospheric boundary layer height and greatly underestimate the Arctic amplification effect on wind speed. Furthermore, we conducted a comparative analysis of three wind power development metrics among four future scenarios to assess the effects of the CMIP models' underestimation on China's 2030 carbon emission reduction target. According to the results, planning the development of wind power in China by using the underestimated CMIP model predictions would considerably hinder China from meeting its carbon peak emission target by 2030 and will delay the 2060 goal of carbon neutrality. These findings emphasize that the CMIP models urgently need to be improved.

This research provides an important scientific basis for a warning to countries throughout the Northern Hemisphere, that wind power development plans should be formulated with consideration of global climate change and its meteorological impacts. Overall, these insights should be of great significance for achieving global carbon 
neutrality.

\section{Methods}

\section{Calculation of the annual average wind speed at a height of $80 \mathrm{~m}$}

The wind speed at each observation station is recorded at a height of $10 \mathrm{~m}$, but the hub height of a wind turbine is generally $80 \mathrm{~m}$. Therefore, the wind speed at a height of $10 \mathrm{~m}$ is extrapolated to a height of $80 \mathrm{~m}$ using a power law relation (a power law coefficient of $1 / 7$ is chosen, but the power law coefficient has been shown to only slightly impact the wind energy potential $\left.{ }^{23}\right)$ :

$$
V_{80 m}=V_{10 m}\left(\frac{80 m}{10 m}\right)^{\frac{1}{7}}
$$

The observations were acquired every 6 hours, but after taking the annual average of the data series, the data adopt a yearly resolution.

The original CMIP6 wind speed data are also represented at a $10 \mathrm{~m}$ height and are also extrapolated to $80 \mathrm{~m}$ using the power law wind profile above (Equation 2). The original temporal resolution is monthly, but after annual averaging, the resolution also becomes yearly. The CMIP6 wind speed data are spatially gridded. Thus, to obtain CMIP6 data spatially coinciding with the 771 observation stations, we perform bilinear interpolation for the CMIP data on the grids adjacent to each observation station. Finally, the CMIP data corresponding to the 771 stations are averaged to compute the linear declining trend for each CMIP model and each SSP scenario (see Supplementary Tables $2-4)$.

\section{Theoretical analysis of the long-term trend of wind speed at a height of $80 \mathrm{~m}$}


For the high-altitude free atmosphere (that is, the atmosphere above the atmospheric boundary layer), the geostrophic Coriolis force is at equilibrium with the pressure gradient force, yielding the following geostrophic wind relationships ${ }^{24-25}$ :

$$
\begin{aligned}
& u_{g}=\frac{1}{f \rho} \frac{\partial P}{\partial y} \\
& v_{g}=-\frac{1}{f \rho} \frac{\partial P}{\partial x}
\end{aligned}
$$

where $u_{g}, v_{g}, P, f$, and $\rho$ are the zonal geostrophic wind, meridional geostrophic wind, air pressure, geostrophic parameter, and air density, respectively.

For the atmosphere under the atmospheric boundary layer, the geostrophic Coriolis force, turbulent friction force, and pressure gradient force are balanced, and the relationship is as follows ${ }^{24-25}$ :

$$
\begin{gathered}
K_{m} \frac{d^{2} \overline{u_{z}}}{d z^{2}}=-f \overline{v_{z}} \\
K_{m} \frac{d^{2} \overline{v_{z}}}{d z^{2}}=f \overline{u_{z}}-f u_{g}
\end{gathered}
$$

where $\overline{u_{z}}$ and $\overline{v_{z}}$ are the average zonal wind and average meridional wind at a height $z$ (under the atmospheric boundary layer, such as at the $80 \mathrm{~m}$ height of a wind turbine hub), respectively, and $K_{m}$ is the turbulent friction coefficient.

Based on the above relationship between the free atmosphere and boundary layer, the ideal gas state equation, the vertical declining temperature gradient, the boundary layer height $H$, and the reasonable simplification of secondary items (using a Boussinesq approximation ${ }^{26}$, etc.), the theoretical relationship of the long-term change in the wind speed at a height of $80 \mathrm{~m} V_{80 \mathrm{~m}}$ is derived as follows:

$$
V_{80 m}=-\frac{R}{f} \frac{\partial T_{2 m}}{\partial y}\left[1+e^{-\frac{160 \pi}{H}}-2 e^{-\frac{80 \pi}{H}} \cos \left(\frac{80 \pi}{H}\right)\right]^{\frac{1}{2}}
$$

The detailed derivation of Equation 7 and the method for calculating the wind speed 
based on ERA5 data are shown in the Supplementary Information.

Method for estimating the average wind speed in 2014 employed to generate wind power in China

Here, the Rayleigh distribution, which is commonly used for the initial estimation of wind energy resources, is employed as the universal statistical wind speed distribution ${ }^{27}$. In the Rayleigh distribution, the distribution function $f\left(v, v_{\text {ave }}\right)$ is related only to the average wind speed $v_{\text {ave }}$ and the wind speed $v$, namely,

$$
f\left(v, v_{\text {ave }}\right)=\frac{\pi v}{2 v_{\text {ave }}^{2}} \cdot \exp \left[-\pi\left(\frac{v}{2 v_{\text {ave }}}\right)^{2}\right]
$$

A typical Rayleigh distribution under $v_{\text {ave }}$ equals $5 \mathrm{~m} / \mathrm{s}$ is shown in Figure 6 a.

For the power curve, we refer to the $2 \mathrm{MW}$ Goldwind GW-115 wind turbine, which is commonly used in Chinese wind farms. Its theoretical power curve $P(v)$ is plotted in Figure $6 \mathrm{~b}$, and its rated power is recorded as $p_{0}$.

a
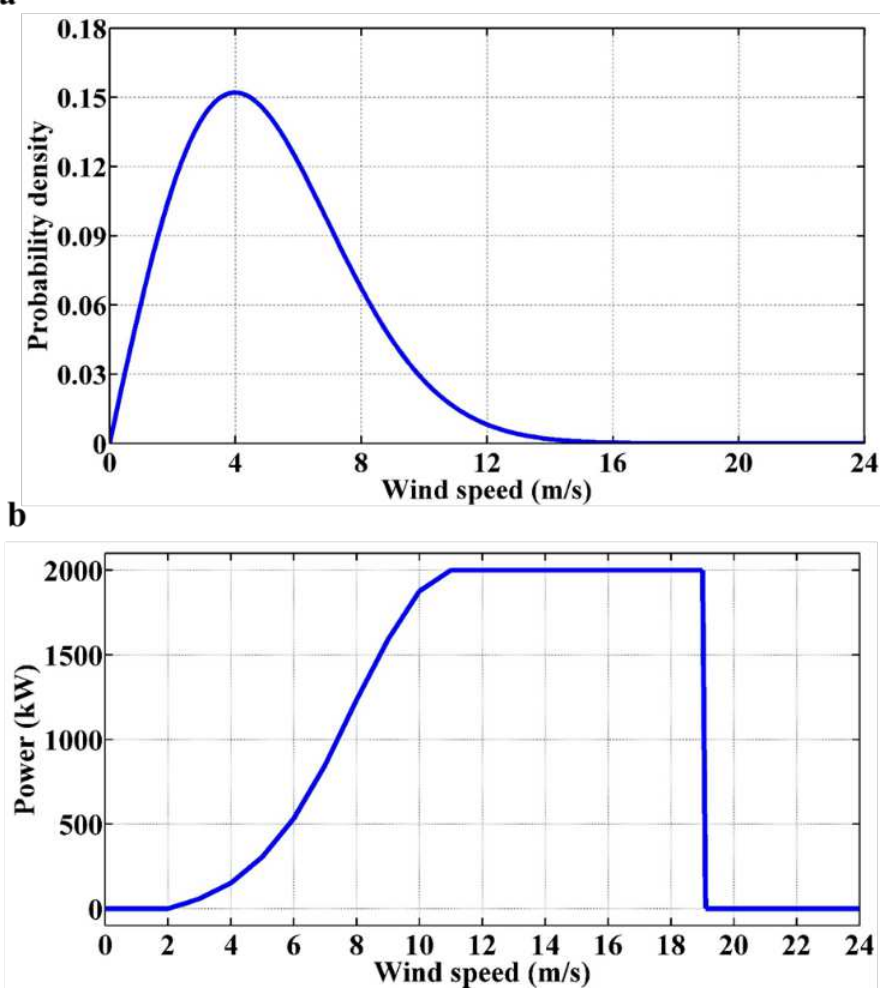
Figure 6 | The probability density distribution of wind speed and power curve used in estimating the average wind speed in 2014 employed to generate wind power in China. a, The probability density of Rayleigh distribution under $v_{\text {ave }}=$ $5 \mathrm{~m} / \mathrm{s}$. b, Theoretical power curve of a $2 \mathrm{MW}$ Goldwind GW-115 wind turbine.

Determining the annual power generated by a wind turbine is equivalent to integrating $P(v)$ with all wind speed distribution intervals over all times of the year. Consequently, the power generated by a wind turbine $W_{0}$ is

$$
W_{0}=\int_{0}^{T}\left[\int_{v=0}^{\infty} P(v) \cdot f\left(v, v_{\text {ave }, 2014}\right) d v\right] d t
$$

where $T$ equals $8760 \mathrm{~h}$ for a year. In 2014, China's total installed wind power capacity $P_{2014}$ was $9.64 \times 10^{7} \mathrm{~kW}$, and the total wind-generated electricity $W_{2014}$ was $1.67 \times 10^{11} \mathrm{kWh}^{28}$. The number of $2 \mathrm{MW}$ wind turbines is computed as $P_{2014} / p_{0}$. Thus, the power generation $W_{2014}$ of all China's wind turbines in 2014 is calculated as

$$
W_{2014}=\left(P_{2014} / p_{0}\right) \cdot W_{0}=\left(P_{2014} / p_{0}\right) \cdot \int_{0}^{T}\left[\int_{v=0}^{\infty} P(v) \cdot f\left(v, v_{\text {ave }, 2014}\right) d v\right] d t
$$

The only unknown quantity in Equation 10 is $v_{\text {ave,2014 }}$, that is, the average wind speed employed to generate wind power in China in 2014; this quantity is obtained based on numerical discrete differentiation. The $v_{\text {ave,2014 }}$ value we calculated is $4.578 \mathrm{~m} / \mathrm{s}$. Thus, to calculate the actual rate of decline $k_{\text {real }}$ of the wind speed $v_{\text {ave,2014 }}$ in China, we select the data from all 771 observation stations and identify the stations in 2014 with an annual average wind speed within $1 \mathrm{~m} / \mathrm{s}$ of the above calculated value $(4.578 \pm 1 \mathrm{~m} / \mathrm{s})$. Ultimately, 69 stations are selected, and their average rate of decline $k_{\text {real }}$ is $-0.3155 \mathrm{~m} / \mathrm{s} / 10 \mathrm{yr}$ based on linear regression. The distribution information and average wind speed of 69 stations are detailed in the Supplementary Information. 


\section{Analysis of China's new installed capacity, new investment costs and new land} occupation in 2030

First, we calculate the annual average wind speed $v_{\text {ave,2030 }}$ employed to generate wind power in China in 2030 under the 4 scenarios as follows:

$$
v_{\text {ave }, 2030}=\left\{\begin{array}{c}
v_{\text {ave }, 2014}-k_{S S p 126} \cdot \frac{(2030-2014)}{10} \text { for SSP126 } \\
v_{\text {ave }, 2014}-k_{S S p 245} \cdot \frac{(2030-2014)}{10} \text { for SSP245 } \\
v_{\text {ave }, 2014}-k_{S S p 585} \cdot \frac{(2030-2014)}{10} \text { for SSP } 585 \\
v_{\text {ave }, 2014}-k_{\text {real }} \cdot \frac{(2030-2014)}{10} \text { for the real data }
\end{array}\right.
$$

where $k_{\text {real }}$ is the observed linear rate of decline $(-0.3155 \mathrm{~m} / \mathrm{s} / 10 \mathrm{yr})$, and $k_{s s p 126}, k_{s s p 245}$, and $k_{s s p 585}$ are the average rates of decline of the three SSP scenarios $(-0.0015 \mathrm{~m} / \mathrm{s} / 10 \mathrm{yr},-0.0037 \mathrm{~m} / \mathrm{s} / 10 \mathrm{yr}$, and $-0.0059 \mathrm{~m} / \mathrm{s} / 10 \mathrm{yr}$ for SSP126, SSP 245 , and SSP585, respectively; see Supplementary Tables 2-4).

After calculating $v_{\text {ave,2030 }}$ under each of the 4 scenarios, we calculate China's 2030 installed capacity $P_{2030}$ under those 4 scenarios as follows:

$$
W_{2030}=\left(P_{2030} / p_{0}\right) \cdot \int_{0}^{T}\left[\int_{v=0}^{\infty} P(v) \cdot f\left(v, v_{\text {ave, } 2030}\right) d v\right] d t
$$

where $T$ equals $8760 \mathrm{~h}$ for a year and the planned wind-generated power $W_{2030}$ is $1.425 \times 10^{12} \mathrm{kWh}$, which considers that $50 \%$ of all power is generated by non-hydro renewable energy according to China's 2030 plan $^{4}$. Since the right side of Equation 12 is already known, China's 2030 installed capacity $W_{2030}$ can be obtained. Then, China's new installed capacity is compared with the capacity in $2020\left(2.8 \times 10^{8} \mathrm{~kW}^{5}\right)$.

After obtaining China's 2030 new installed capacity, the new investment costs and the new area of land occupation are similarly calculated. We assume that the installed capacity increases with a uniform increment each year. The cost is based on the global 
average cost of 1,473 USD/kW in 2019 (including wind turbine and installation costs), and the cost decreases by $5 \%$ annually ${ }^{29}$. For the land area occupied, according to China national standards ${ }^{30}$, the row spacing (spacing parallel to the main wind direction) between wind turbines is 5 times the diameter of the rotor, and the row spacing (spacing perpendicular to the main wind direction) is 3 times the diameter of the rotor. We again refer to the $2 \mathrm{MW}$ Goldwind GW-115 wind turbine, and the diameter of the rotor is taken as $115 \mathrm{~m}$. Then, a total installed capacity of 100,000 kW (the installed capacity of 50 wind turbines) is divided among 10 columns and 5 rows, which is equivalent to an area of $9.92 \mathrm{~km}^{2}$.

\section{References}

[1] Future of wind: Deployment, investment, technology, grid integration and socioeconomic aspects (International Renewable Energy Agency, 2019).

[2] Global Wind Report 2021 (Global Wind Energy Council, 2021).

[3] Speech at the General Debate of the Seventy-fifth United Nations General Assembly by Xi Jinping. (The State Council of the People's Republic of China, 2020).

[4] Letter on Soliciting the Responsibility Weight of Renewable Energy Electricity Consumption in 2021 and the Proposals for the Expected Target from 2022 to 2030 (National Energy Administration of China, 2021) (in Chinese).

[5] 2020 National Electric Power Industry Statistics Express List (National Energy Administration of China, 2021) (in Chinese).

[6] Karnauskas, K. B., Lundquist, J. K. \& Zhang, L. Southward shift of the global wind 
energy resource under high carbon dioxide emissions. Nat. Geosci. 11, 38-43 (2017). [7] Meehl, G. A. et al. Overview of the Coupled Model Intercomparison Project. Bull. Amer. Meteor. Soc. 86, 89-93 (2015).

[8] Simpkins, G. Progress in climate modelling. Nat. Clim. Chang. 7, 684-685 (2017). [9] Meehl, G. A. et al. The WCRP CMIP3 multi-model dataset: A new era in climate change research. Bull. Amer. Meteor. Soc. 88, 1383-1394 (2007).

[10] Rowlands, D. J. et al. Broad range of 2050 warming from an observationally constrained large climate model ensemble. Nat. Geosci. 5, 256-260 (2012).

[11] Carvalho, D., Rocha, A., Gómez-Gesteira, M. \& Santos, C. S. Potential impacts of climate change on European wind energy resource under the CMIP5 future climate projections. Renew. Energ. 101, 29-40 (2017).

[12] Wu, J., Shi, Y. \& Xu, Y. Evaluation and projection of surface wind speed over China based on CMIP6 GCMs. J. Geophys. Res. 125, e2020JD033611 (2020).

[13] Sherman, P. et al. Projected changes in wind power potential over China and India in high resolution climate models. Environ. Res. Lett. 16, 034057 (2021).

[14] Knutti, R. \& Sedláček, J. Robustness and uncertainties in the new CMIP5 climate model projections. Nat. Clim. Chang. 3, 369-373 (2013).

[15] Climate Change 2014: Synthesis Report (Intergovernmental Panel on Climate Change, 2014)

[16] AR6 Climate Change 2021: The Physical Science Basis (Intergovernmental Panel on Climate Change, 2021).

[17] Masson-Delmotte, V. et al. Past and future polar amplification of climate change: 
climate model intercomparisons and ice-core constraints. Clim. Dyn. 26, 513-529 (2006).

[18] Polyakov, I. V. et al. Observationally based assessment of polar amplification of global warming. Geo. Res. Lett. 29, 25-1-25-4 (2002).

[19] Cohen, J. et al. Linking Arctic variability and change with extreme winter weather in the United States. Science 373, 1116-1121 (2021).

[20] Hersbach, H. et al. The ERA5 global reanalysis. Q. J. R. Meteorol. Soc. 146, 1999$2049(2020)$

[21] Eyring, V. et al. Overview of the Coupled Model Intercomparison Project Phase 6 (CMIP6) experimental design and organization. Geosci. Model Dev. 9, 1937-1958 (2016).

[22] O'Neill, B. C. et al. A new scenario framework for climate change research: the concept of shared socioeconomic pathways. Clim. Change 122, 387-400 (2014).

[23] Walter, K. et al. Speed and direction shear in the stable nocturnal boundary layer. J. Sol. Energy Eng. 131, 011013 (2009).

[24] Sheng, P. X. Atmospheric Physics (Peking Univ. Press, 2013) (in Chinese).

[25] Stull, R. B. An Introduction to Boundary Layer Meteorology (Springer Science \& Business Media, 1988).

[26] Zeytounian, R. K. Joseph Boussinesq and his approximation: a contemporary view. C. R. - Mec. 331, 575-586 (2003).

[27] Wind turbines - Part 1: Design Requirements IEC 61400-1 4th edition (International Electrotechnical Commission, 2019). 
[28] Monitoring Situation of the Wind Power Industry in 2014 (National Energy Administration of China, 2015) (in Chinese).

[29] Renewable Power Generation Costs in 2019 (International Renewable Energy Agency, 2020).

[30] Technical code for Micro-siting of Wind Power Projects NB/T 10103-2018 (China Energy Industry Standard, 2018) (in Chinese).

\section{Acknowledgements}

This work is supported by the Joint Research Fund in Smart Grid (Grant No. U1966601) under cooperative agreement between the National Natural Science Foundation of China (NSFC) and State Grid Corporation of China(SGCC).

\section{Author contributions}

Shuanglei Feng and Weisheng Wang designed and directed the research. Shuanglei Feng designed and conducted the calculating of wind power development scale under different future scenarios. Zongpeng Song designed and conducted the theoretical research of wind speed decline, and processed the data of observation and CMIP. Qing Yang, Feng Liu, and Yunhe Hou analysed the data of observation and CMIP. All authors contributed to developing and writing the manuscript.

\section{Competing interests}


The authors declare no competing interests. 


\section{Supplementary Files}

This is a list of supplementary files associated with this preprint. Click to download.

- SupplementaryInformationSeverelyunderestimatedrateofwindspeeddeclinejeopardizesChinascarbonpeakemissiontarget.docx 